\title{
Analysis of Three CRISPR Loci Among Yersinia pestis Stains from Georgia and Neighboring Countries
}

\section{Ekaterine Zhghenti, Gvantsa Chanturia}

molecular biology and genomic research, NATIONAL CENTER FOR DISEASE CONTROL AND PUBLIC HEALTH, Tbilisi, Georgia

Objective

The purpose of our study was CRISPR-based analysis of $Y$. pestis isolates from Georgia and neighboring countries.

Introduction

Particular family of tandem repeats, such as Clustered Regularly Interspaced Short Palindromic Repeats (CRISPR) found in a wide range of prokaryotic genomes. CRISPRs consist of highly conserved repeats interspaced with non-repetitive elements or "spacers" usually of viral origin. In the Yersinia pestis genome, three CRISPR elements YPa, YPb and YPc are found. The distribution of spacers and their arrays in Y. pestis strains is region and focus specific and can provide important information for genotyping and evolutionary research of bacteria.

\section{Methods}

Whole-genome sequencing (WGS) of 14 Y. pestis strains from Georgia, Armenia, Azerbaijan, Russian Federation (RF) and Kirgizstan were performed using a combination of different next generation sequencing (NGS) platforms: Illumina, PacBio and 454 technologies. Identification of spacer sequences from CRISPR loci was conducted to evaluate the genetic diversity among the isolates. The spacers arrays were acquired and analyzed. DRs and spacers were identified and extracted. The selected spacer sequences were compared against the microbial genome database in GenBank. Leader sequences were obtained for each CRISPR locus and aligned using MAFFT software.

\section{Results}

In all 14 sequenced genomes, three CRISPR elements were found. All alleles show the same organization, conserved $28 \mathrm{bp}$ repeated sequence is interspaced with spacers, 31 or $34 \mathrm{bp}$ in length. A total of 18 spacers were found: seven for the YP1, six for the YP2 and five forYP3. According to the spacer arrays sequence, Biovar Microtus strains from Georgia and Armenia had the same allele sizes with the presence of seven motifs, for YP1 (a1b1c1d1e1m1n1), six motifs for YP2 (a2j2b2k212m2) and five for YP3 loci (a3b3c3d3e3) identical to PestoidesG (genotype 1). Spacers/spacer arrays of Biovar Medievalis strains from Georgia, Azerbaijen and Kabardino Balkaria (RF) were the same as KIM (genotype - 59).

\section{Conclusions}

CRISPR analysis of Georgian strains identified two independent phylogenetic groups that is in agreement with previous study data (SNP typing, IS100 fingerprinting). Biovar Microrus strains from Transcaucasian highland placed within one of the most ancient branches of the evolutionary model of $Y$. pestis. This study enhances our understanding and fulfills the existing data of genetic characteristics of $Y$. pestis strains circulating in the region. 